\title{
General Psychiatry Where is the global in global mental health? A call for inclusive multicultural collaboration
}

\author{
Tom L Osborn (D) , ${ }^{1,2}$ Akash R Wasil, ${ }^{3}$ John R Weisz, ${ }^{1}$ Arthur Kleinman,,${ }^{4,5}$ \\ David M Ndetei ${ }^{6,7}$
}

To cite: Osborn TL, Wasil AR, Weisz JR, et al. Where is the global in global mental health? A call for inclusive multicultural collaboration. General Psychiatry 2020;33:e100351. doi:10.1136/ gpsych-2020-100351

Received 21 July 2020 Revised 14 September 2020 Accepted 22 September 2020

Check for updates

(C) Author(s) (or their employer(s)) 2020. Re-use permitted under CC BY-NC. No commercial re-use. See rights and permissions. Published by BMJ.

${ }^{1}$ Department of Psychology, Harvard University, Cambridge, Massachusetts, USA

${ }^{2}$ Shamiri Institute, Nairobi, Kenya ${ }^{3}$ Department of Psychology, University of Pennsylvania, Philadelphia, Pennsylvania, USA ${ }^{4}$ Department of Anthropology, Harvard University, Cambridge, Massachusetts, USA

${ }^{5}$ Department of Global Health and Social Medicine, Harvard Medical School, Boston,

Massachusetts, USA

${ }^{6}$ Africa Mental Health Research and Training Foundation, Nairobi, Kenya

${ }^{7}$ Department of Psychiatry, University of Nairobi, Nairobi, Kenya

Correspondence to

Tom L Osborn;

tomleeosborn@gmail.com
To the editor:

Common mental health disorders, like depression and anxiety, account for $45 \%$ of the global disease burden on youths aged 15-29. ${ }^{1}$ This challenge is particularly significant in low and middle-income countries (LMICs) where risk factors such as poverty, limited treatment options and stigma restrict access to care and exacerbate the burden and magnify the impact of mental disorders. ${ }^{2}{ }^{3}$ Given these barriers, there is an urgent need for research dedicated to expanding mental healthcare for young people in LMICs.

Because of the global public health challenge characterized by adolescent mental health disorders, there have been increased efforts over the last two decades to expand treatment options for youths in LMICs. These efforts-conducted under the umbrella of global mental health research-have improved our understanding of the efficacy and acceptability of various kinds of mental health interventions for young people in LMICs. However, there are concerns that these efforts may risk the imposition of Western values and approaches. Researchers from high-income countries (HICs) may bring assumptions about diagnostic classification, aetiology and treatment that may not be appropriate for LMICs. ${ }^{4}$ To address this risk, and enhance cultural validity, some investigators have emphasised the value of collaborating with colleagues from LMICs in designing and implementing interventions for these settings. ${ }^{5}$ Without such collaboration with community partners, well-intentioned efforts may risk relying on false assumptions about the generalisability of Western-derived intervention development and research methods across diverse populations in LMICs. ${ }^{4}$

A possible consequence of the status quo is that mental health interventions in LMICs may fail to adequately consider and address salient features of adolescent mental health in LMICs. Indeed, scholars have noted several unique obstacles that investigators face when deploying interventions in LMICs. These include a paucity of mental health experts, differences in help-seeking attitudes and cultural factors that can affect the acceptability and appropriateness of mental health interventions. ${ }^{6}$ Furthermore, Western researchers often bring treatments that are aimed at targeting mental disorders as defined by the Diagnostic and Statistical Manual of Mental Disorders (DSM) or the International Statistical Classification of Diseases and Related Health Problems (ICD) taxonomies. However, the experience and expression of mental disorders is shaped by culture, which has been shown to influence the aetiology, the neurobiology and the manifestation of mental disorders. ${ }^{7}$ In fact, a recent study in Kenya found that the DSM-5 classification for major depressive disorder did not capture salient features of this disorder within a community sample in Kenya. ${ }^{8}$ Thus, simply transporting Western treatments to non-Western settings may not be ideal; treatments that recognise and address the local complexities of the experience of mental illness are needed .

With these concerns in mind, we sought to understand the extent to which intervention research for adolescents has actively involved input from people in LMICs. We reviewed a recent database of 15 randomised controlled trials (RCTs) testing various psychological treatments for common adolescent mental health disorders trialled in the last two decades with 8421 adolescents in LMICs. ${ }^{9}$ The authors of the database conducted a literature search using PubMed and Google Scholar for RCTs of depression, anxiety and post-traumatic stress disorder in children and adolescents in LMICs that were published 
up to 2017 (see the review for more information ${ }^{9}$ ). We wanted to investigate the extent to which these efforts involved multicultural collaboration with researchers and other experts located within the LMICs where the studies were conducted. We operationalised multicultural collaboration in two ways: (A) the extent to which the first authors on the RCT papers were affiliated with an institution based in an LMIC, and (B) the proportion of all the authors in the RCT papers who were affiliated with an institution based in an LMIC. Besides this, we also considered-in the cases in which the first author was affiliated with an LMIC-based institution-whether the author's affiliation was from the same LMIC population as that being studied. For example, were the authors from Nigeria while the study is in Pakistan?

We found that only 5 (33.33\%) of the 15 first authors were affiliated with an institution based in an LMIC. All of the five first authors were affiliated with institutions from the same population being served. We also found that only $31(34.44 \%)$ of the 90 authors in these RCTs were affiliated with an institution based in an LMIC.

Though treatment intervention research in LMICs is increasingly collaborative, the research efforts seem to be driven primarily by researchers based in HICs. The limited number of first authors affiliated with an LMIC institution also suggests that local researchers and experts are not primarily responsible for driving research efforts in LMIC settings. This status quo is unfortunate because treatment interventions in LMICs can benefit from the active inclusion of researchers and experts from LMICs in the intervention development process. ${ }^{5}$

One way in which adolescent mental health research done in LMICs can benefit from the expertise of local researchers may lie in the local sociocultural expertise that researchers from LMICs bring to the table. This local expertise helps intervention development efforts overcome some of the local barriers that limit help-seeking or influence the treatment of adolescent mental illness. One such local barrier is stigma. Stigma influences how youths experience mental health disorders and whether they seek treatment for these disorders. ${ }^{10}$ Even though the concept of stigma may be universal, its manifestation is particularly localised and cultural. For example, crosscultural research has revealed that societal stigma leads Chinese patients to highlight the somatic symptoms of mental health problems rather than the affective ones. ${ }^{4}$ Experts from LMICs are more attuned to the sociocultural nuance in LMIC settings and can guide intervention development efforts to circumvent societal stigma and other LMIC-specific barriers that prevent youths from seeking mental healthcare. ${ }^{11}$

Another reason for increasing the involvement of LMIC researchers in global mental health research is that they may improve the acceptability and eventual adoption of treatment intervention in LMICs. One challenge that intervention development efforts derived by HIC researchers may face-and one that is particularly pervasive in the international development space but is also relevant to global mental health research-is that these interventions may be viewed as top-down interventions by locals in LMICs. As such, their acceptability and adoption may be watered down. The inclusion of LMIC researchers in intervention development efforts may lend local credibility to mental health interventions in LMICs and increase their adoption. ${ }^{12}$

Change that privileges the inclusion of LMIC researchers and experts in global mental health research is needed and feasible. Such change may also be particularly important as we respond to the global mental health challenges that have arisen from the COVID-19 pandemic. ${ }^{1314}$ Beyond simple collaboration with LMIC research institutions, researchers from LMICs should be empowered and involved throughout the research process. LMIC researchers should be involved to the extent that their roles meet professional criteria for authorship, including as first or last authors where warranted by the extent and nature of their contributions. Beyond working with local researchers and experts, collaboration with community members should also be encouraged and sought. Local community members bring their lived experiences, which enrich the research process, and offer insights into the target audience. A benefit of including community members in the research process is the development of socioculturally acceptable treatments that will truly expand access to mental healthcare. A worthy goal should be that community members are centrally involved in the research process and in ways that meet criteria for co-authorship in publications. Research funders, and journal editors, should consider setting up mechanisms that encourage the authors of RCTs in LMICs to seek and prioritise multicultural collaboration with experts and community members.

\section{Twitter Tom L 0sborn @tomleeosborn}

Contributors TLO and ARW conceived this manuscript. TLO, ARW, JRW, AK and DN participated in the drafting and revising of the manuscript.

Funding The authors have not declared a specific grant for this research from any funding agency in the public, commercial or not-for-profit sectors.

Competing interests None declared.

Patient consent for publication Not required.

Provenance and peer review Not commissioned; externally peer reviewed.

Open access This is an open access article distributed in accordance with the Creative Commons Attribution Non Commercial (CC BY-NC 4.0) license, which permits others to distribute, remix, adapt, build upon this work non-commercially, and license their derivative works on different terms, provided the original work is properly cited, appropriate credit is given, any changes made indicated, and the use is non-commercial. See: http://creativecommons.org/licenses/by-nc/4.0/.

ORCID iD

Tom L Osborn http://orcid.org/0000-0002-9092-1035

\section{REFERENCES}

1 The Lancet. Better understanding of youth mental health. Lancet 2017;389:1670.

2 Patel V, Flisher AJ, Hetrick S, et al. Mental health of young people: a global public-health challenge. Lancet 2007;369:1302-13.

3 Patel V, Kleinman A. Poverty and common mental disorders in developing countries. Bull World Health Organ 2003;81:609-15. 
4 Kleinman A. Rethinking psychiatry. Simon and Schuster, 2008.

5 Verdeli H. Global mental health: an introduction. J Clin Psychol 2016;72:761-5.

6 Patel V, Chowdhary N, Rahman A, et al. Improving access to psychological treatments: lessons from developing countries. Behav Res Ther 2011;49:523-8.

7 Shattuck EC. A biocultural approach to psychiatric illnesses. Psychopharmacology 2019;236:2923-36.

8 Osborn TL, Kleinman A, Weisz JR. Complementing standard Western measures of depression with locally Co-developed instruments: a cross-cultural study on the experience of depression among the Luo in Kenya. Transcultural Psychiatry 2020.

9 Yatham S, Sivathasan S, Yoon R, et al. Depression, anxiety, and post-traumatic stress disorder among youth in low and middle income countries: a review of prevalence and treatment interventions. Asian J Psychiatr 2018;38:78-91.
10 Ndetei DM, Mutiso V, Maraj A, et al. Stigmatizing attitudes toward mental illness among primary school children in Kenya. Soc Psychiatry Psychiatr Epidemiol 2016;51:73-80.

11 Osborn TL, Wasil AR, Venturo-Conerly KE, et al. Group intervention for adolescent anxiety and depression: outcomes of a randomized trial with adolescents in Kenya. Behav Ther 2020;51:601-15.

12 Osborn TL, Wasanga CM. Simple, Stigma-free, scalable, and schoolbased: a four-step approach to developing adolescent mental health treatments in sub Saharan Africa. PsyArXiv, 2020. Available: https:// psyarxiv.com/apku2/

13 Qiu J, Shen B, Zhao M, et al. A nationwide survey of psychological distress among Chinese people in the COVID-19 epidemic: implications and policy recommendations. Gen Psych 2020;33:e100213.

14 Gruber J, Prinstein MJ, Clark LA, et al. Mental health and clinical psychological science in the time of COVID-19: challenges, opportunities, and a call to action. Am Psychol 2020. doi:10.1037/ amp0000707. [Epub ahead of print: 10 Aug 2020].

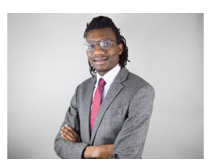

Tom L. Osborn completed a bachelor's degree (high honors) in Psychology in 2020 from Harvard University, Cambridge, USA. Since 2020, he has been working as the Founder and Executive Director of Shamiri Institute, Inc, a nonprofit organization whose mission is to develop and deploy evidence-based psychological interventions that improve the mental health and well-being of young people in low and middle-income countries such as his home country of Kenya. Besides his work at Shamiri Institute, he is currently a research associate at the Moral Psychology Research Lab in the Department of Psychology at Harvard University. His main research interests include developing accessible mental health interventions for at-risk youths in low-income settings, adolescent psychopathology across Sub-Saharan Africa, psychological interventions that improve educational outcomes, and social learning mechanisms. 